\title{
РЕКОМЕНДАТЕЛЬНЫЙ СЕРВИС ДЛЯ ТОРГОВЛИ АКЦИЯМИ НА ФОНДОВОМ РЬНКЕ С ПРИМЕНЕНИЕМ МАШИННОГО ОБУЧЕНИЯ
}

\section{RECOMMENDATION SERVICE FOR TRADING SHARES ON THE STOCK MARKET USING MACHINE LEARNING \\ G. Semyannikov}

Summary. In the modern world, globalization and the development of information technologies dictate the need to implement various computer technologies in all spheres of public relations, based on the use of artificial intelligence methods. One such method is machine learning, which is the ability of computers to learn new things autonomously. In recent years, machine learning has not only become a major financial industry, but also took the first place among the technologies used on the back-end markets. The more data is processed using machine learning, the more accurate the conclusions obtained using such training. Machine learning allows you to facilitate decision-making processes by analyzing and identifying significant patterns, directing traders to a specific decision based on forecasted indicators.

The author analyzes the possibility of using machine learning technologies in stock markets and demonstrates how machine learning algorithms are implemented when trading shares on the stock market.

Keywords: stock markets, stocks, machine learning, investment, price forecasting, deep learning, benchmark.
Семянников Глеб Валерьевич

Национальный исследовательский технологический университет «Московский институт стали и сплавов», институт Информационных бизнес-систем g.l.e.b.s@yandex.ru

Аннотация. В современном мире глобализация и развитие информационных технологий диктуют необходимость внедрения во все сферы общественных отношений различных компьютерных технологий, в основе использования которых лежат методы искусственного интеллекта. Одним из таких методов является машинное обучение, представляющее собой способность компьютеров изучать новые вещи автономно. В последние годы машинное обучение не только стало основой финансовой отрасли, но и заняло первое место среди технологий, используемых на фоновых рынках. Чем больше данных обрабатывается с помощью машинного обучения, тем точнее выводы, полученные с применением такого обучения. Машинное обучение позволяет облегчить процессы принятия решений, анализируя и выявляя существенные закономерности, направляя трейдеров к определенному решению, основанному на прогнозируемых показателях.

Автором статьи анализируется возможность использования технологий машинного обучения на фондовых рынках и демонстрируется, как алгоритмы машинного обучения реализуются при торговле акциями на фондовом рынке.

Ключевые слова: фондовые рынки, акции, машинное обучение, инвестиции, прогнозирование цен, глубокое обучение, бенчмарк.
B современном мире машинное обучение не ограничивается только техническими средствами, используемыми в повседневной жизни. В последние годы машинное обучение стало опорой в финансовой отрасли, а особенно часто оно стало применяться на фондовом рынке [6].

В наиболее общем понимании машинное обучение представляет собой способность компьютеров изучать новые вещи автономно $[2,5]$. Процесс обучения основан на данных, прошлом опыте и наблюдениях. Чем больше данных обрабатывает компьютер, тем лучше он делает выводы. И именно поэтому алгоритмы машинного обучения стали неотъемлемой частью ДНК финансовых рынков.
Однако возникает объективный вопрос о том, как именно инвестиции в акции выигрывают от прогресса в области машинного обучения? Сегодня торговый процесс эволюционировал массово до состояния, когда трейдеры используют сложные параметры и комбинации факторов для того, чтобы прийти к итоговому решению. При этом осуществляется в том числе и оценка социальных настроений через технические индикаторы. Инвестирование сегодня сложнее, чем когда-либо, а машинное обучение может облегчить весь этот процесс, анализируя большие куски данных, выявляя существенные закономерности и создавая один выход, который направляет трейдеров к определенному решению, основанному на прогнозируемых ценах активов. 
Предсказать, как будет работать фондовый рынок,это одна из самых сложных задач трейдера. Объясняется это тем, что трейдеру для прогнозирования ситуации на фондовом рынке необходимо учитывать множество факторов: физических, физиологических, рациональное и иррациональное поведение и т.д. Все эти факторы объединяются и анализируются для определения постоянно изменяющихся цен на акции, которые очень трудно предсказать с высокой степенью точности. Методы машинного обучения имеют потенциал для обнаружения паттернов и идей, которые трейдеры не учитывали ранее, однако которые могут быть использованы для безошибочно точных прогнозов.

Существенное значение использования алгоритмов машинного обучения для прогнозирования фондового рынка отмечается в работах российских и иностранных исследователей. Так, например, специалисты школы компьютерных наук Николы Милошевича Манчестерского университета доказали, что машинное обучение позволяет осуществлять эффективное прогнозирование долгосрочного движения цен на акции [9]. Группа ученых из Стенфорда на примере использования алгоритмов машинного обучения для прогнозирования больших американских фондовых индексов, в том числе таких индексов, как Dow Jones Industrial Average, доказала, что уровень точности прогнозов при использовании машинного обучения может достигать 77,6\% [10]. П.А. Абаньшин и М.Ю.Барышникова, в свою очередь, акцентируют внимание на том, что при использовании текстового майнинга для анализа новостного потока и оценки оттенка новостей, возможно составить эффективный прогноз поведения ценных бумаг на фондовом рынке. Преимуществом такого метода является его ориентация на факторы, которые не учитываются традиционными методами экономики и финансов [1, с. 24-27]. А.А. Майорский при этом отмечает, что использование таких технологий машинного обучения, как нейросети, аналитика больших данных (Bigdata) и облачные вычисления в биржевой торговле позволяет усовершенствовать современные стратегии трейдинга за счет получения более точных данных при прогнозировании [3, с. 229-233].

Стоит согласиться со всеми вышеприведенными позициями. Прогнозирование и моделирование играет важную роль в анализе данных фондовых рынков. В своей основе фондовые рынки, как правило, непредсказуемы и даже нелогичны, при этом они испытывают зависимости от различных факторов, в частности таких, как результаты голосования в Великобритании по Brexit, цены на нефть марки Brent, результаты последних выборов в США, карантин в европейских странах в связи с COVID-2019. В силу этих особенностей финансовые данные следует считать обладающими довольно хаотич- ной структурой, что часто затрудняет поиск устойчивых закономерностей. Чтобы решить эту проблему, алгоритм должен быть снабжен как можно большим количеством объективной информации.

Моделирование хаотических структур требует алгоритмов машинного обучения, способных находить скрытые законы в структуре данных и предсказывать, как они повлияют на нее в будущем. Наиболее эффективной методологией для достижения этой цели является «глубокое обучение». Глубокое обучение позволяет легко справляться со сложными структурами и извлекать взаимосвязи, которые еще больше повышают точность получаемых результатов.

Теперь проанализируем, как на практике осуществляется построение моделей глубокого обучения. Подход машинного обучения в биржевой торговле не сильно отличается от подхода, который обычно используют аналитики-трейдеры. Первым шагом является организация набора данных для предпочтительного инструмента. Затем он делится на две основные группы: обучающий набор и тестовый набор. Прежде чем алгоритм будет протестирован, он должен быть обучен и точно настроен, а для этого необходим обучающий набор. После того, как становится ясно, что алгоритм соответствует всем требованиям, он вводится в действие с помощью тестового набора. После того, как алгоритм генерирует результат, он сравнивается с реальной производительностью конкретного запаса.

Теперь рассмотрим пример логики, лежащей в основе алгоритма машинного обучения для биржевой торговли. Существует множество способов построения прогностического алгоритма. Однако большинство из них обычно следуют определенной логике: сбор необходимых данных, построение алгоритма, интерпретация результатов, поскольку это простой и эффективный способ для базовых прогнозов фондового рынка.

Как мы уже отмечали выше, финансовые рынки - это хаотические структуры. А хаотические процессы показывают, что события прошлого могут оказывать огромное влияние на настоящее и будущее. Это делает исторические данные хорошим источником для прогнозирования будущих цен на финансовые инструменты.

Если сделать предположение о том, что основное внимание уделяется торговле акциями, то, прежде всего, трейдер должен выяснить, какие инструменты его интересуют, а также загрузить и подготовить соответствующие исторические данные в формате временных рядов. Далее трейдеру следует выбрать бенчмарк, чтобы он мог сравнить результаты работы алгоритма с его производительностью. Так, если взять, например, 
Таблица 1. Оценки топ-50 акций, отфильтрованных по степени предсказуемости

\begin{tabular}{|c|c|c|c|c|c|c|}
\hline \multirow{2}{*}{$\begin{array}{l}\text { топ- } 50 \\
\text { прогнозируемых }\end{array}$} & \multicolumn{6}{|c|}{ Эффект сигнала } \\
\hline & три дня & Одна неделя & Две недели & Один месяц & Три месяца & Год \\
\hline топ-30 сигналов & $0,21 \%$ & $0,35 \%$ & $0,67 \%$ & $1,35 \%$ & $4,04 \%$ & $19,25 \%$ \\
\hline топ-20 сигналов & $0,25 \%$ & $0,37 \%$ & $0,69 \%$ & $1,21 \%$ & $4,06 \%$ & $19,63 \%$ \\
\hline топ-10 сигналов & $0,22 \%$ & $0,38 \%$ & $0,81 \%$ & $1,33 \%$ & $4,58 \%$ & $21,95 \%$ \\
\hline топ-5 сигналов & $0,24 \%$ & $0,52 \%$ & $1,19 \%$ & $1,67 \%$ & $4,96 \%$ & $22,78 \%$ \\
\hline бенчмарк & $0,13 \%$ & $0,23 \%$ & $0,50 \%$ & $1,07 \%$ & $3,09 \%$ & $14,63 \%$ \\
\hline превосходство & $60,46 \%$ & $51,94 \%$ & $34,49 \%$ & $26,03 \%$ & $30,71 \%$ & $31,61 \%$ \\
\hline
\end{tabular}

индекс Standard \& Poor's 500 (S\&P 500), то необходимо установить временные рамки, в течение которых будет проанализирована производительность индекса. Возьмем данные за 3-летний период с января 2016 года по январь 2019 года.

Следующим шагом является построение алгоритма. При этом основная цель - построить алгоритм, способный прогнозировать траектории движения цен. Хороший способ достичь этого - стремиться к двум основным факторам: сигналу и предсказуемости. Идея первого заключается в том, чтобы представить, что такое ожидаемое движение (вне зависимости от того, что это будет - повышение цены или ее снижение). Фактор предсказуемости предназначен для выявления корреляции между прошлыми прогнозами алгоритма и реальным движением каждого из наблюдаемых активов. Или, другими словами, чтобы показать, насколько уверенными являются результаты для сигнала. Для получения более точных результатов трейдеры используют коэффициент корреляции Пирсона.

Оценки проводятся на уровне отдельных запасов, а затем усредняются. Доходность каждой сделки рассчитывается по следующей формуле:

$$
\text { доходность }=\frac{\text { конечная цена }}{\text { цена в день прогноза }-1}
$$

Для упрощения, вычисления следует производить в Excel. Результат из приведенной выше формулы показывает, какова ожидаемая доходность, если инвестор покупает инструмент в день прогноза и продает его через 1 месяц. Это открывает путь для поиска лучших инструментов для торговли из всего индекса. Следующий шаг состоит в том, чтобы дать указание алгоритму взять среднее значение всех прогнозов и взвесить.

Последний, третий этап — это интерпретация результатов. После того, как алгоритм машинного обучения закончит свои прогнозы, трейдер должен отфильтровать наиболее предсказуемые инструменты в списке и выбрать те, которые имеют наибольшую силу сигнала. Таким образом, он может легко определить, какие акции наиболее вероятно испытают ценовое движение. Далее трейдер должен сравнить результаты от наиболее высокоэффективных акций с соответствующим бенчмарком и принять инвестиционное решение. В таблице представлен образец оценки топ - 50 акций, отфильтрованных по степени предсказуемости (таблица 1).

Из табличных данных видно, что алгоритм превосходит доходность индекса S\&P 500 по всем инвестиционным горизонтам.

Еще одной эффективной методологией является «ансамблевое обучение». Идея, лежащая в его основе, заключается в использовании мощности нескольких алгоритмов обучения для повышения общей точности окончательного прогноза. Преимущество такой методологии заключается в том, что процесс обучения и алгоритм обучения происходят независимо друг от друга. После их завершения сгенерированные прогнозы объединяются в один обобщенный анализ. Два из наиболее популярных алгоритмов, которые часто объединяются с помощью ансамблевого обучения - это нейронные сети и машины опорных векторов.

Вне зависимости от того, какие алгоритмы машинного обучения будут использованы, можно точно утверждать, что в настоящий момент использование методов машинного обучения и искусственного интеллекта для прогнозирования цен на акции является растущей тенденцией. Все больше и больше исследователей работают над тем, чтобы разработать оптимальные алгоритмы, которые помогут еще больше повысить точность модели прогнозирования цен на акции.

Из-за огромного количества доступных опционов, может быть много способов, как предсказать цену акции, но все методы не работают одинаково. Выходные данные различаются для каждого метода, даже если применяется один и тот же набор данных. В рамках настоящей статьи автором акцентировалось внимание на прогнозировании цены акций с использованием алгоритма «глубокое обучение», который используется для прогнозирования цены акций с использованием фи- 
нансовых коэффициентов за предыдущие периоды. Это лишь один из способов взглянуть на проблему оценки на фондовом рынке, приблизившись к ней с помощью прогностической модели. Однако, как уже было отмечено, всегда есть и другие факторы, влияющие на цену акций, такие, как настроения инвестора, общественное мнение о компании, новости от различных торговых бирж и события, которые заставляют весь фондовый рынок колебаться. Поэтому, по мнению автора статьи, при использовании машинного обучения на фондовых рынках необходимо выбирать такие алгоритмы машинного обучения, которые не только являются самыми простыми и гибкими в использовании, но и дают хорошую точность в прогнозировании цен на акции с учетом анализа настроений на рынках.
Резюмируя вышесказанное, необходимо отметить, что прогнозирование цен на акции еще десять лет назад было обширным и трудоемким процессом. Сегодня сила алгоритмов машинного обучения помогает сохранить время и усилия при торговле акциями на фондовом рынке. Однако, этой технологии еще предстоит пройти долгий путь, пока она не станет полностью способной решить основную «загадку» финансовых рынков. И именно поэтому алгоритмы машинного обучения стали неотъемлемой частью ДНК финансовых рынков. Машинное обучение может облегчить весь процесс, анализируя большие куски данных, выявляя существенные закономерности и создавая один выход, который направляет трейдеров к определенному решению, основанному на прогнозируемых ценах активов.

\section{ЛИТЕРАТУРА}

1. Абаньшин П.А., Барышникова М. Ю. Сравнительный анализ методов автоматизированного прогнозирования изменения цен акций на фондовом рынке. Вестник науки и образования. — 2020.— № 3(81).— Часть 1.— С. 24-27.

2. Лобин М.А., Филиппова И. А. Машинное обучение в экономике. Вестник Ульяновского государственного технического университета. - 2019. - № 4 (88). - C. 68-70.

3. Майорский А. А. Машинное обучение в биржевой торговле. Е-Scio.— 2019. — № 10 (37). - С. 229-233.

4. Федонова Г.В., Ботнарь С. Ю. Особенности алгоритмической торговли на фондовом рынке // Теория и практика сервиса: экономика, социальная сфера, технологии. - 2016. № 3 (19). - С. 11-14.

5. Филиппов Д. И. Особенности развития финансового рынка Европейского союза в условиях глобализации. — М., 2014. — 227 с.

6. Филиппов Д. И. Финансовые инновации в процессе трансформации цифровой экономики. Вестник Российского экономического университета имени Г. В. Плеханова.— 2018. - № 3.- С. 58-71.

7. Филиппов Д.И. Теория и методология оценки влияния финансовых инноваций на развитие финансового рынка / Монография.- М.: ФГБОУ ВО «РЭу им. Г. В. Плеханова», 2019. - 380 с.

8. Флах П. Машинное обучение. Наука и искусство построения алгоритмов, которые извлекают знания из данных. -М.: ДМК Пресс, 2015. - 402 с.

9. Equity forecast: Predicting long term stock price movement using machine learning, Nikola Milosevic School of Computer Science, University of Manchester, 2016. [Электронный ресурс]. Режим доступа: https://arxiv.org/pdf/1603.00751.pdf/ (дата обращения: 23.03.2020).

10. Shunrong Shen, Haomiao Jiang and Tongda Zhang. Stock market forecasting using machine learning algorithms, Stanford, 2012. [Электронный ресурс]. Режим доступа: http://cs229.stanford.edu/proj2012/ShenJiangZhangStockMarketForecastingusingMachinelearningalgorithms.pdf/ (дата 0бращения: 23.03.2020). 